\title{
PROJECT FLEXIBILITY AND LEAN CONSTRUCTION
}

\author{
Glenn Ballard ${ }^{1}$ and Hajnalka Vaagen ${ }^{2}$
}

\begin{abstract}
The Last Planner system was initially focused only on production control functions, trying to improve the match between SHOULD and CAN, CAN and WILL, and WILL and DID. A partial specification of SHOULD was introduced into Last Planner practice in 1998 with the pull planning of phase schedules. The next logical extension of Last Planner is to the planning of master schedules and the project execution strategies of which they are a part. One of the key elements in that extension is managing variation, both negative and positive (risks and opportunities). This paper offers a framework for managing variation. Subsequent papers will apply that framework in processes for producing project execution strategies and master schedules.
\end{abstract}

Keywords: Lean construction, Uncertainty, Flexibility.

\section{INTRODUCTION AND A FRAMEWORK TO HANDLE VARIATION IN PROJECTS}

Kahneman \& Tversky (1982) distinguish between internal and external randomness, in order to differentiate between phenomena for which it is potentially wise to collect information to reduce the variation, and phenomena for which this makes no sense. In the context of project based production systems, that distinction appears as two types of variation: 1) Statistically describable variation, for which the mitigation strategy is a) variation reduction and $b$ ) buffering the 'at-that-time' irreducible variation; and 2) Low probability/high impact changes, for which creating flexibility, to adapt changes with least disturbance, is the appropriate strategy. Buffering would be prohibitively costly in time or money, and would not exploit the opportunities that are offered by such events. See e.g. Wallace (2010) for a more detailed discussion.

This paper applies two industry cases to identify and exemplify the above distinction, and offers a framework for managing variation in projects. Subsequent papers will apply that framework for producing project execution strategies and master schedules. The research foundation was established by a series of incremental steps undertaken through long term research engagement within the Aera Energy case for oil field development, and a shipbuilder for advanced marine operations.

The Aera Energy case illustrates how to manage variation that is statistically describable; including both how to reduce variation and how to buffer not-yet-reducible variation. These methods were applied to an oil field development process conceived as a multi-project processing system in which individual wells were the projects.

The shipbuilding case, with the Last Planner system (Ballard and Tommelein 2016) partially implemented, discusses design changes, where the frequency of occurrence is

1 Dr. Ballard is Research Director for the Project Production Systems Laboratory at the University of California Berkeley. ballard@ce.berkeley.edu

2 Dr. Vaagen is Associate Professor at the Norwegian University of Science and Technology, dept. of Ocean Operations and Civil Engineering. Hajnalka.vaagen@ntnu.no 
well known but the type of change is difficult to anticipate. This type of variation, along with concurrency in engineering and execution to reduce lead times, dictates a complex planning problem where the project network itself is altered (i.e. the tasks to be performed and their sequencing), not only the task durations. See Vaagen et al. (2017) for the technical aspects of the problem. The social aspects of an observed proactive behaviour, to handle low probability/high impact events, are discussed in Vaagen et al. (2016).

The proposed framework to manage variation in projects is illustrated by Figure 1. It consists in identification of the two major types of variation, and strategies to manage them. In Section 2 and Section 3 examples are provided for statistically describable variation and buffering strategies, then for low probability/high impact events and flexibility strategies. Conclusion and future research paths are given in Section 4.

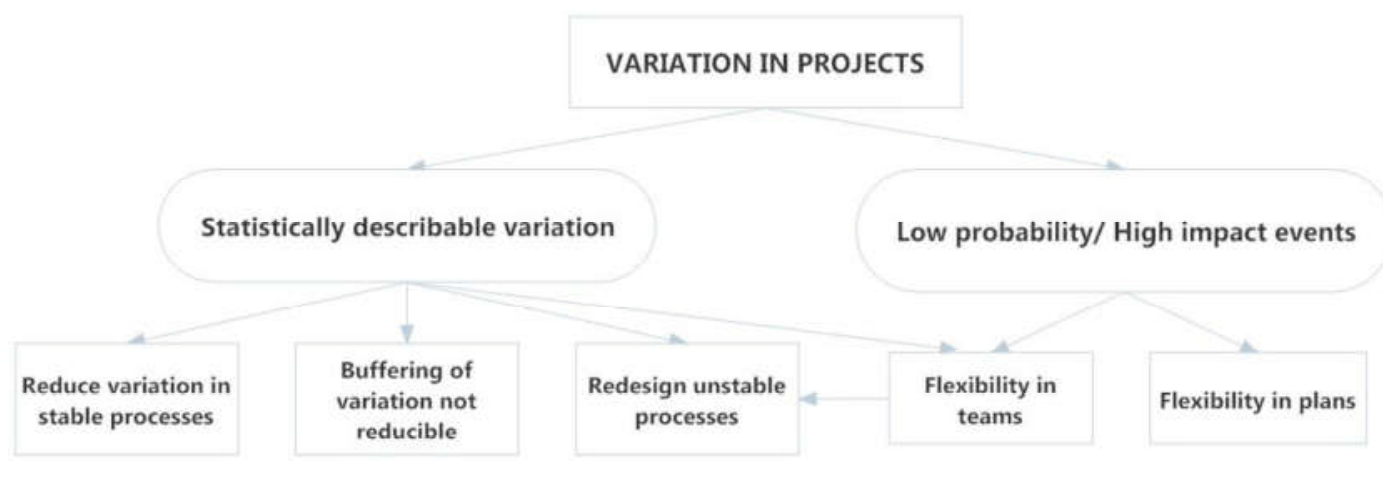

Figure $1 \mathrm{~A}$ framework to manage variation in projects

\section{Statistically Describable VARIATION}

Processes may be stable or unstable. This distinction is the basis for statistical process control (Leavenworth and Grant, 2000) and the basis for the lean advice to first stabilize, then improve processes (Liker, 2004). Stable processes have statistically predictable variation resulting from the nature of the process; i.e., from so-called 'common' causes. By definition, the variation in stable processes cannot be reduced by identifying special causes/disturbances to the process; the process itself must be redesigned.

The variation in processing durations of unstable processes can be reduced by identifying 'special' causes of variation and implementing countermeasures.

It is accepted that variation, though potentially reducible, is unavoidable in some degree at any point in time, and consequently that buffers are needed in all production systems. There are three types of buffers: time, inventory and capacity (Hopp and Spearman, 2011). There are also three production system design choices: type of buffer, location of the buffer and sizing of the buffer.

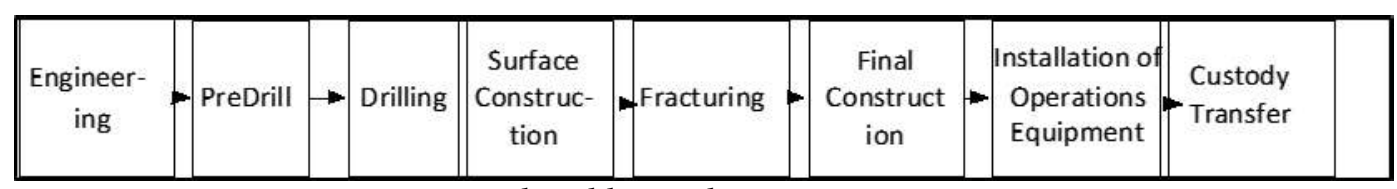

Figure 2 Oil Field Development Process 
All of these elements can be seen in the Aera Energy case. When the first author began working with them in 2000, Aera Energy, already on its lean journey, asked for help applying lean to oil field development, which extends from engineering design of wells to custody turnover of the producing well to operations-as shown in Figure 2.

\subsection{Reducing variation}

Oil field development can be understood as a multi-project production system, in which each well is a project. In the Aera Energy case, three types of changes were made to reduce variation: 1) Reduction of batch sizes, 2) Right sizing equipment, and 3) Improving estimates of process durations.

A study of the system identified large batch sizes as a driver of wasted capacity and unnecessarily long cycle times. In an attempt to reduce the penalty imposed by setup times, the number of wells fractured from a single location had increased to as many as 50. The processes that followed Fracturing had increased their crews and equipment in an attempt to handle the 50 wells, which resulted in idle capacity during the time required for fracturing and releasing a new batch. Fracturing's batches were the first to be reduced in size, from the previous norm of 50 wells to a maximum of 12 .

Another batch size problem was discovered in the packages of wells released into the process, which had grown to match the transfer batch of 50 being released by Fracturing. This batch was also reduced to a maximum of 12 and Engineering was asked to select those 12 wells for an average processing duration approximately equal to the takt time for the system, which was calculated to be 2.1 wells/day.

Since the size of transfer batches are the primary driver of cycle time (Hopp and Spearman, 2011), this reduction in batch size contributed to reducing cycle time by $36 \%$. Having the takt target enabled better capacity allocation and provided a criterion for deciding when accelerating a well was needed. That took more variation out of the development process.

The large Fracturing batches originated in efforts to reduce the amount of time required to disassemble, move and reassemble fracturing equipment (shown in Figure 3 following) in a new location. The setup time penalty was larger than necessary because the fracturing equipment was not right-sized for the job. Aera Energy's light oil development consisted of wells approximately 2000 feet deep, but the fracturing equipment being used, shown in Figure 3, was designed for much deeper wells. The fracturing batch was reduced to a maximum of 12, and the equipment was right-sized.

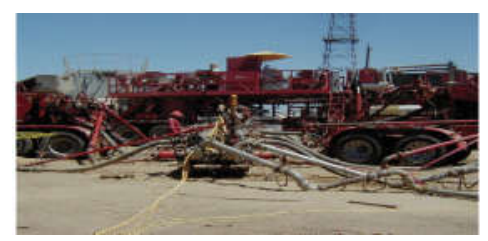

Figure 3 Fracturing Equipment

Prior to the intervention described here, the duration of drilling each well was estimated by the Drilling Engineer. These estimates were used to forecast the release of wells from Drilling to Fracturing. In an attempt to improve those forecasts, a study was done comparing the Driller and Drilling Engineer's duration estimates. It was found that, after only one day (typically $25 \%$ of drilling duration), the Driller could more accurately forecast completion time of drilling. 


\subsection{Restructuring the system}

Once the system was stabilized, it was restructured (see Figure 4 following). Changes were made to the organizational structure, a production control system was introduced, and both inventory and capacity buffers were built into the oil development process.

Value Stream Managers were appointed for each of the process boxes shown in Figure 2 previously, and the Last Planner System (Ballard and Tommelein, 2016) was introduced as a means for coordinating between processes. As found in applications of the Last Planner in other industry sectors (e.g., Vitaliy and Sacks, 2015), it not only improved reliability of work flow as a result of Value Stream Managers proactive coordination and planning, but also increased flexibility of response to the occasional plan failures, which reduced the amount of inventory and capacity needed in the respective buffers. This flexibility in plans and in teams is discussed in more detail in Section 3.

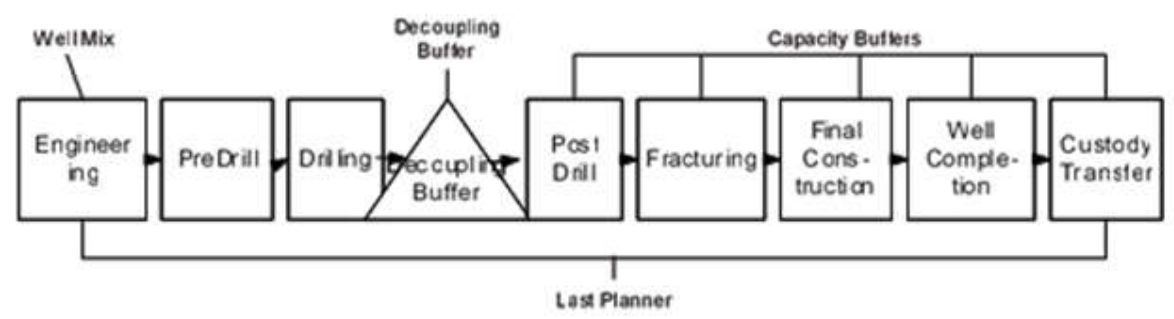

Figure 4 Production control mechanisms

A decoupling buffer was then created after drilling to release wells pulled from Fracturing. This level loaded the processes that followed Drilling, but just in case of need, overtime was reserved as a capacity buffer. The results of these initiatives to reduce variation, then buffer remaining variation were a reduction in capacity cost throughout the oil field development process of $25 \%$ and a $36 \%$ reduction in cycle time.

\section{Low PRobability/High IMPACT EvEnTS}

These events may pose risks or opportunities for project success. They typically originate from outside the project, are difficult to predict and, therefore, difficult to manage. Examples include changes in regulations, in market demand, in market conditions (competitors' actions, exchange rates, political risk). In specialized shipbuilding, design changes driven by regulations and market demand are well known, but the type of change is difficult to anticipate. It may be everything, from changes in door/ window positions (changing piping and electro systems), to large scale outfitting solutions coming late into the project execution (like the demand for helicopter deck or firefighter system), altering the project network itself by changing the scope.

The appropriate strategy for low probability/high impact events is to create flexibility in plans (King and Wallace, 2012, Vaagen et al., 2017), and flexibility in teams to quickly adapt to- and absorb variations and disturbances (also known as one capability of resilient systems; see e.g. Hollnagel et al. 2006). These are discussed below.

\subsection{Flexibility}

Flexibility as an operations objective refers to the firm's ability to change or adapt its operations to provide flexibility of mix (product or service), product flexibility, volume 
flexibility and delivery time flexibility (Slack et al, 2007). A flexible system is one with options, where future decisions are conditioned on the arrival of new and relevant information. Strategies with options have higher initial costs than those without options (i.e. creating flexibility is usually not free), but enable adaptation to a different future scenario with least disturbance. Vaagen et al. (2017) report up to 35\% lower costs of proactive (flexible) strategies as compared to deterministic reactive plans, where an initial optimal plan, developed to fit one certain design, is updated when new information arrives. A flexible strategy not only adapts to changes, but anticipates them and is prepared to react to them.

\subsubsection{Flexibility in plans}

Flexibility in plans can be achieved by a number of strategies, usually as a combination of hedging and postponement.

Postponement refers to decisions to be postponed to/and updated when new (and relevant) information becomes available, and when it is also feasible to update the decisions. Examples are given below.

Structuring the work to increase knowledge about stakeholders in the project definition process to better inform decisions (see. e.g. Mok and Shen, 2016).

Keep the solution space open and plan in greater detail as the work being planned approaches nearer in time (see e.g. Emblemsvåg, 2014).

Apply the decouple point concept, to develop 'two-stage planning processes'. Activities that can be executed with optimized workflow, with the information available, are assigned to the first stage. Customization activities are assigned to the second stage, after learning more about the future. The decouple point defines the degree of customization, and is the driving force behind the development of a modular design strategy, combining the advantages of standardization and customization in the same value delivery process (Robertson and Ulrich, 1998).

Hedging refers to developing or buying an 'insurance' to offset potential losses or gains. Examples are:

Set-based design to develop a fallback alternative design in case it is needed to meet the Last Responsible Moment (Ward, et al., 1995).

By consolidating negatively correlated activities, flexibility and free hedging can be achieved (King and Wallace, 2012). The general behaviour of correlations is that negative correlations reduce risk and are perceived as hedging in financial and product portfolio planning, while positive correlations increase risk. Planning flexibility has, therefore, low value in case of positive correlations, with planned buffers suggested as solution by Lium et al. (2009). Negatively correlated activities are observed when, e.g., design is uncertain, and one alternative potentially excludes others (may be because of weight \& space restrictions). Uncertain activities competing for the same resources (e.g. equipment or space) are other sources of negative correlations; see Schuyler (2001) for more. Assessing correlations to develop prioritization rules and understand which activities to plan in parallel or sequentially, would significantly affect project risk. 
The case below exemplifies postponement and hedging in specialized shipbuilding. Here, foundations for outfitting equipment are increasingly incorporated into the steel structure of the hull units. Good solution from a design perspective, but at high production costs because production of units must wait until foundations are determined, and changes of foundations also automatically lead to extra production costs. Postponement -by removing foundations from the steel units, and placing them in during the unit outfitting stage, when outfitting details are known- provides greater production flexibility, lower costs, and shorter time to market. A hedging initiative is to deck the entire prow with thicker steel sheets regardless of outfitting. Figure 5 illustrates the 'inflexible' situation with exact placement of thicker plates according to outfitting details, with great amount of rework time reported to adjust changes in outfitting details.

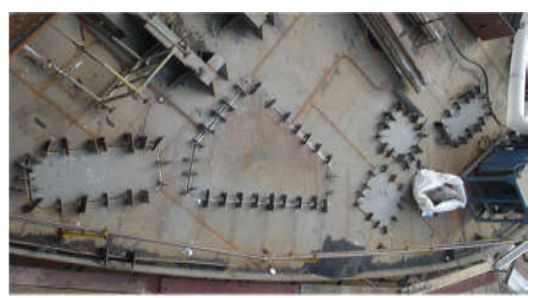

Figure 5 Illustration of outfitting equipment foundations in shipbuilding

Note that hedges need not be used in order to perform their function of preserving possible future events. For example, both the fallback alternative and the thicker steel sheets may not be needed. They are a type of insurance that enable the possibility to adopt changes. We tend to prefer not to have to use our insurance. In this respect, hedges differ from buffers, which are built into the production system, and are consumed in performing their function of absorbing variation in e.g. task duration.

\subsubsection{Flexibility of Teams}

Team flexibility is understood in the sense that team members recognize, adapt to- and absorb variations and disturbances, especially those that are not anticipated. The shortcomings of model-based approaches to handle low probability/ high impact changes, switched focus to the social network characteristics that facilitate a proactive behavior to cope with disturbance under pressure (Vaagen et al., 2016). Vitaliy and Sacks $(2013,2015)$ found that projects on which Last Planner was used strengthened their social network. Further, there have been numerous unpublished reports that project teams using Last Planner are better able to adapt to changes. A possible explanation is that teams and team members become habituated and skilled in developing plans to achieve objectives in uncertain and variable circumstances. We might call this a mindset of "planning to complete', as distinct from looking back to an original plan to assess project progress.

The above suggest that Last Planner facilitates the development of responsive teams. Concretely, constraint analysis forces teams to evaluate alternative outcomes and solutions under incomplete information, while reliable commitment forces teams to actually make a decision; i.e., to make a "more-or-less intelligent" guess, postpone the decision, develop hedging or assign buffers. This, in order, leads to the understanding that team flexibility can be further improved by building the social network that can exploit options in plans and develop options not in the plan. Further research is needed here, to better understand how to apply Last Planner to explore these aspects, to help people proactively cope with disturbance under pressure. 


\section{CONCLUSION AND FUTURE RESEARCH}

This paper provides a framework for managing variation in projects, to be applied to the development of project execution strategies and the master schedules contained in them. The framework is based on classifying variation is projects as statistically describable and low probability/high impact events. While the first type usually describes variation in task completion time for a given project network, the latter may significantly alter the project network itself. Methods for managing statistically describable variation include redesign of unstable processes, reduction of variation in stable processes, and buffering of variation not yet reducible. Methods for managing low probability/high impact events focus on creating flexibility in plans by postponement and hedging strategies, and by facilitating team responsiveness. These are summarized on Figure 1.

Although the suggested strategies are well established, the way they are applied and combined depends on how different frameworks distinguish between the types of randomness. For example, Husby et al. (1999) differentiates uncertainty along the project life cycle, and suggests mitigation actions based on the influence possibility throughout this timeline. Our framework is driven by acknowledging uncertainty that alters the project network itself, which also makes PERT planning methods less useful. At the same time, we acknowledge that the Last Planner system handles, to a certain extent, some of the non-anticipated changes. Which leads to the motivation to apply the suggested framework to the development of project execution strategies and master schedules within them, within the theoretical frame of Last Planner. To better understand this, consider the distinction between risks and opportunities, as the differentiation between cost- and profit-driver external uncertainties. Customer driven design change provides value with a market price for change; i.e. opportunity. This is to be treated differently from changes that are primarily cost drivers (e.g. in legal and environmental regulation). Analyzing the structural properties of the profit- and costdriver uncertainties: the first one is a profit maximization problem, while the second is a cost minimization problem. It is obvious that, on higher planning levels, these require distinct solution approaches (and distinct project networks). However, once the options are 'paid', the problem turns to cost-focus objectives. Which suggests that the differentiation between profit- and cost-driver external uncertainties only relates to the project execution strategy and master plans, where the options that enable flexibility are to be treated. On lower levels the problem structure is similar: changes and errors are to be handled there-and-then, with least disturbance. This is where great improvements from Last Planner implementation are already reported, while the mechanisms, by which the project network itself is altered, are less understood.

\section{ACKNOWLEDGMENT}

The authors thank Professor Stein Wallace, Norwegian School of Economics, for valuable discussions.

\section{REFERENCES}

Ballard, Glenn and Iris D. Tommelein (2016). Current Process Benchmark for the Last Planner System. Project Production Systems Laboratory, University of California Berkeley. Available at p2sl.berkeley.edu. 
Emblemsvåg, J., 2014. Lean project planning in shipbuilding. Journal of Ship Production and Design. 30 (2), 79-88.

Leavenworth, Richard S., and Eugene Lodewick Grant. Statistical quality control. Tata McGraw-Hill Education, 2000.

Hollnagel, E., Woods, D. D. \& Leveson, N. C. (Eds.) (2006). Resilience engineering: Concepts and precepts. Aldershot, UK: Ashgate.

Hopp, Wallace J., and Mark L. Spearman. Factory physics. Waveland Press, 2011. 127, 239-251.

Kahneman, D., Tversky, A. (1982). Judgment under uncertainty: Heuristics and biases. (Kahneman, D., Slovic, P., \& Tversky, A., Eds.). Cambridge, UK: Cambridge University Press.

King, A. J., Wallace, S. W., 2012. Modeling with Stochastic Programming. Springer Series in Operations Research and Financial Engineering. Springer.

Lium, A.G., Crainic, T.G., Wallace, S.W., 2009. A study of demand stochasticity in stochastic network design. Transportation Science 43(2), 144-157.

Liker, Jeffrey K. The Toyota way. Esensi, 2004.

Mok, M.K. and Shen, G.Q., 2016. A network-theory Based Model for Stakeholder Analysis in Major Construction Projects, Procedia Engineering, 164, 292-298.

Vitaliy, P., Sacks, R., 2013. Social Network Development in Last Planner System ${ }^{\mathrm{TM}}$ Implementations. Proceedings for the 21st Annual Conference of the International Group for Lean Construction. 2013.

Vitaliy, P., Sacks, R., 2015. Effects of the last planner system on social networks among construction trade crews. Journal of Construction Engineering and Management, 141(6), 04015006.

Robertson, D. and Ulrich, K., 1998. Planning for Product Platforms. Sloan Management Review, 39(4), 19.

Schuyler, J.R.: Risk and Decision Analysis in Projects. Project Management Institute (2001).

Slack, N., Chambers, S., and Johnston, R. (2007). "Operations management", Pearson Education, ISBN 9780 273-70847-6, Fifth edition, Chapter 2.

Vaagen, H., Borgen, E., Hansson, M., 2016. A social-behavioural approach to project work under uncertainty. IFAC-PapersOnLine (ISSN 2405-8963), 49(12), 203-208.

Vaagen, H., Kaut, M., Wallace, S.W., 2017. The impact of design uncertainty on planning. In Press: European Journal of Operational Research. http://dx.doi.org/10.1016/j.ejor.2017.03.005

Wallace, S. W., 2010. Stochastic programming and the option to do it differently. Annals of Operations Research 177 (1), 3- 8.

Ward, A., Liker, J. K., Cristiano, J. J., \& Sobek, D. K. (1995). The second Toyota paradox: How delaying decisions can make better cars faster. Sloan management review, 36(3), 43. 